\title{
TAKING INFINITY BUS IMBALANCE INTO ACCOUNT IN POWER FLOW CALCULATIONS USING NEWTON'S METHOD IN OPTIMIZATION
}

\author{
Andrey Pazderin, Sergey Yuferev ${ }^{a}$ \\ Ural Federal University named after the first President of Russia B.N.Yeltsin, Ural Power Engineering Institute, \\ 620000 Ekaterinburg, Russia
}

\begin{abstract}
This paper studied the influence of the adding infinity bus imbalance to the objective function to be minimized to solve the problem of the power flow calculations by means of Newton's method in optimization. Infinity bus influence on the trajectory of pulling the operation point into existence domain was analyzed by means of a range of the test model calculation performing. It is estimated the infinity bus arguments influence on the trajectory of pulling the operation point into existence domain. A thorough analysis of the test calculation results shows the considerable decreasing the influence of the initial estimates accuracy on the steady-state calculations result.
\end{abstract}

\section{Introduction}

The main method that is used in many power flow calculation programs is Newton's method. This is simple and well-convergence method but it has one big disadvantages: it is impossible to calculate an power flow when operation point determined outside the existence domain. The solving is Newton's method in optimization usage. It can calculate the necessary volume of control actions to pull such point on the border of the existence domain. Due to the low popularity of this method between specialized software developers many aspects of its usage is not studied.

This paper devoted to the question of taking infinity bus imbalance into account in power flow calculations by means of the Newton's method in optimization. It will be shown that such approach can considerably improve convergence of calculation process by means of decreasing the initial estimates accuracy influence on it.

\section{Mathematical Description}

Newton's method in optimization is a second-order method[1]. That function is approximated by Taylor series; it is enough to use only two first elements of the calculated series. For example, for function such expansion is the following:

a Corresponding author: s.v.yuferev@urfu.ru

This is an Open Access article distributed under the terms of the Creative Commons Attribution License 4.0, which permits unrestricted use, distribution, and reproduction in any medium, provided the original work is properly cited. 


$$
\begin{aligned}
& \varphi\left(\Delta X_{1}, \Delta X_{2}\right)=F\left(X_{1}^{(0)}, X_{2}{ }^{(0)}\right)+\Delta X_{1} \frac{\partial F}{\partial X_{1}}+\Delta X_{2} \frac{\partial F}{\partial X_{2}}+ \\
& +\frac{1}{2}\left(\Delta X_{1}^{2} \frac{\partial^{2} F}{\partial X_{1}{ }^{2}}+2 \Delta X_{1} \Delta X_{2} \frac{\partial^{2} F}{\partial X_{1} X_{2}}+\Delta X_{2}^{2} \frac{\partial^{2} F}{\partial X_{2}{ }^{2}}\right)
\end{aligned}
$$

In the common case the criterion function to be minimized determined by the following way[2]:

$$
F=\sum \Delta P^{2}+\sum \Delta Q^{2}
$$

This paper studied grids with the PV buses only. It allows us to remain Jacobian matrix a constant (because of the voltage magnitude invariance) that in turn will prevent existence domain borders changing. In the upshot it simplifies comparison of results obtained by the calculation methods with and without taking infinity bus imbalance into account. The voltage magnitude changing influence on the existence domain of the simplest network (Fig. 1) presented on the Fig. 2.

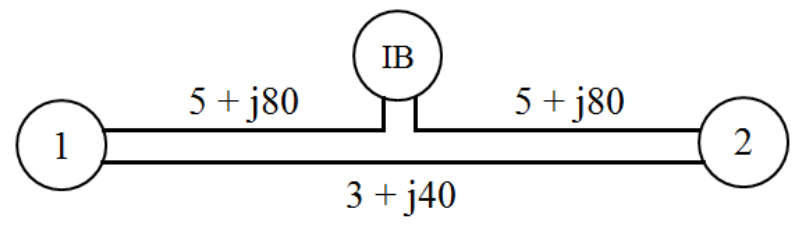

Figure 1. Simplest 3-bus network scheme

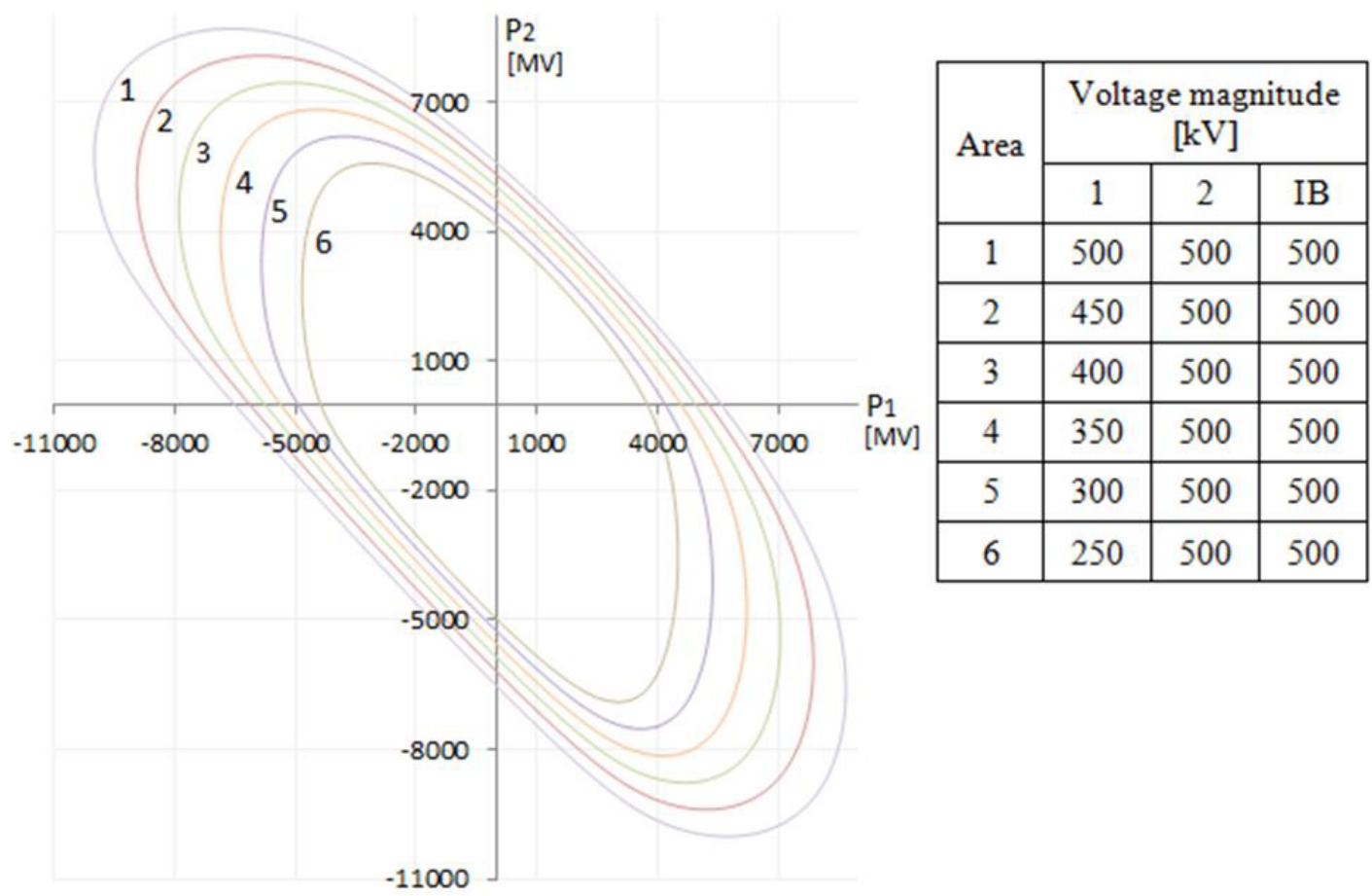

Figure 2. Voltage magnitude changing influence on the existence domain

Thus, it is accepted that on this stage it is possible not to take into account reactive power imbalance in the function to be minimized, and after the infinity bus imbalance adding the function that was presented in Eq. 2 will transform to the following: 


$$
F=\sum \Delta P_{P V}^{2}+\Delta P_{V \delta}^{2}
$$

where the $\sum \Delta P_{P V}^{2}$ is the squared active power imbalance sum of the all PV buses, and the $\Delta P_{V \delta}^{2}$ is a squared imbalance of the infinity bus.

To make a comparison between steady-state calculation results with and without influence bus imbalance in the criterion function it was necessary to realize Newton's method in optimization algorithm, and Matlab development environment was selected. Besides the range of service modules the developed program includes several universal modules that can calculate first- and second-order voltage and phase angle derivatives of the power. Mechanism of the taking influence bus imbalance into account is based on these modules of the Newton's method in optimization algorithm modification.

Firstly, it was necessary to change Hessian and function to be minimized gradient calculation modules. Adding the influence bus imbalance to the criterion function, in fact, removes the influence bus from the power network itself. But it is crucial to save reference bus to avoid appearing of the singular Hessian matrix. Secondly, because of the fact that the total power imbalance in the grid redistributes between all the active buses, final imbalance calculation module also was changed. Finally, to simplify comparison of the test calculation results an additional program module for existence domain border searching was developed.

\section{Algorithm realization and test results}

To illuminate influence bus imbalance influence on the power flow calculation results a range of tests was implemented. The first massive of these calculations consist of "normal" steady-state with the operational point inside the existence domain, the second one - with the operational point outside this domain. The thorough analyzing of the "normal" states calculations shows the necessity of the preliminary network balancing by means of determining the initial power value of the former influence bus as the other buses power values sum. If the balancing is not complete and the power value of the former infinity is zero the power redistribution will be inadequate because of the power source absence. That phenomenon becomes brightly apparent if the operational point determined outside the existence domain, and Fig. 3 shows the difference between calculations of the preliminary balanced and not balanced states in that case.

But even if we balance the initial operational point the result calculated by the modified algorithm will not be on the border of the existence domain (but too close to it) in time the non-modified Newton's method in optimization will. This can be explained by the fact that phase angle values obtained by these methods are a little bit different (voltage magnitude is a constant because of the PV buses); in turn that lead to insignificant change of the existence domain border. 


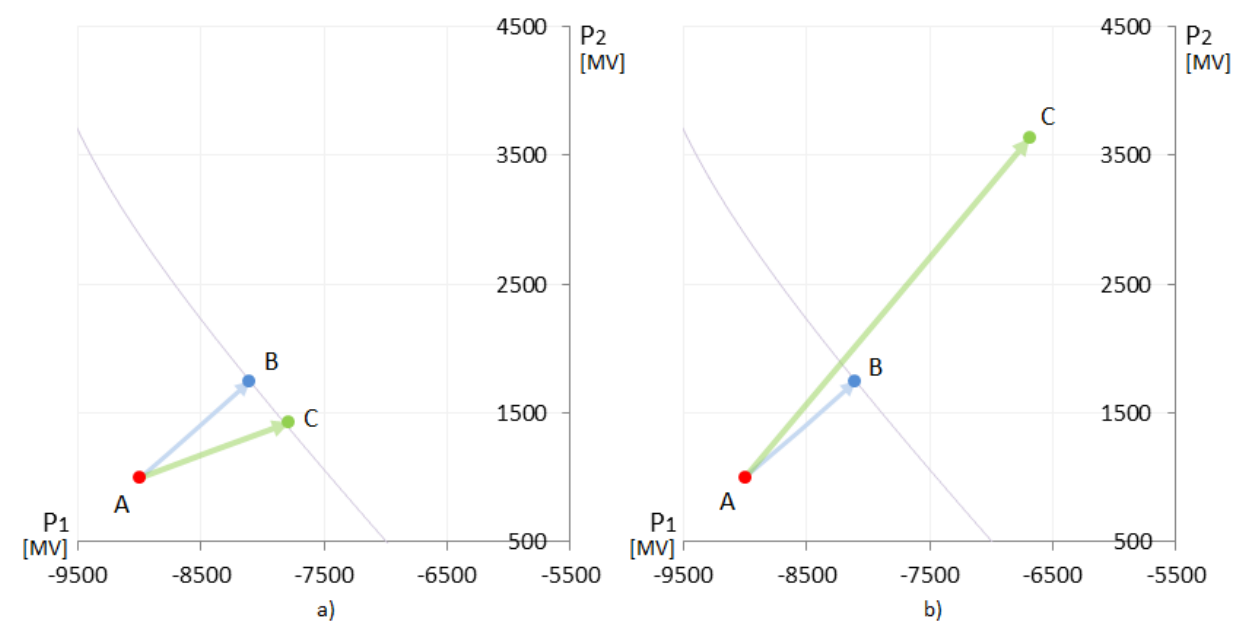

Figure 3. Calculation result of the preliminary balanced (a) and not balanced (b) networks; " $A$ " is an initial operation point outside the existence domain, "B" is the Newton's method in optimization calculation result, point " $C$ " shows the result obtained by the studied algorithm.

It is important to note that the volume of the control action in both cases of steady-state calculations with and without adding infinity bus imbalance to the criterion function is different, and the modified algorithm suggests the bigger actions. That is a negative consequence of the studied method usage; the positive are the following.

Tests show: in most cases a lesser amount of iterations is needed to calculate power flow if modified (with taking infinity bus imbalance into account) algorithm is used. But the more important conclusion is the adding the infinity bus imbalance to the criterion function drastically decreases the influence of the initial estimates accuracy on the result. Thus, condition number of the Hessian matrix on average diminishes in 3 times. In other words taking infinity bus imbalance into account considerable improves exactitude of the power flow calculations.

\section{Conclusion}

According to the experimental results, the following conclusions can be made.

- Power flow calculation by means of Newton's method in optimization with taking infinity bus imbalance into account allows significantly decrease the influence of the initial estimates accuracy on the calculation result.

- It is necessary to continue research of the studied method by adding PQ buses to the model that can permit simulate the real networks behavior, that in turn can help to find the way to decrease the control actions volume needed to put operation point into existence domain that are bigger in the studied method than in the case of non-modified Newton's method in optimization usage.

This work was carried out with the financial supported by Act 211 Government of the Russian Federation, contract № 02.A03.21.0006 and the Ministry of Education and Science of the Russian Federation (in the framework of state assignment, №13.1928.2014/K (project №1928)).

\section{References}

1. M. S. Bazaraa, Hanif D. Sherali, C. M. Shetty, Nonlinear Programming: Theory and Algorithms, 3rd Edition (2006)

2. A. Pazderin, S. Yuferev, Steady-State Calculation of Electrical Power System by the Newton's Method in Optimization” PowerTech (IEEE Bucharest, Romania, 2009) 